\title{
Additional diagnostic testing of the 2019 novel coronavirus (SARS-CoV-2)
}

\author{
Van Giau Vo ${ }^{1,2} \odot$ Eva Bagyinszky ${ }^{1,2} \cdot K_{y}$ uhwan Shim ${ }^{2} \cdot$ Yoon Soo Park ${ }^{3} \cdot$ Seong Soo A. An ${ }^{2}$
}

Received: 16 March 2020 / Accepted: 29 June 2020 / Published online: 7 July 2020

(C) The Korean Society of Toxicogenomics and Toxicoproteomics 2020

\begin{abstract}
Purpose of review Within the last two decades several members of the Coronaviridae family namely Severe Respiratory Syndrome (SARS-CoV) and Middle East Respiratory Syndrome (MERS-CoV) have demonstrated epidemic potential. In late, 2019 an unnamed genetic relative, later named SARS-CoV-2 realized its potential in the highly populous neighborhoods of Wuhan, China. Unchecked, the virus rapidly spread among interconnected communities and related households before containment measures could be in acted. "Appropriate" diagnostic testing in response to the SARS-CoV-2 outbreak should be urgently considered. This perspective review gives particular attention to the potential diagnostic testing of the virus in semen and seminal fluids due to its high levels of angiotensin converting enzyme 2 (ACE2) precursor.

Recent findings As many infectious viruses are stable in semen and have transmitted the respective diseases, the presence of SARS-CoV-2 should be tested in semen to assess their stabilities and half-life. As in case of Ebola virus, it was present in semen for longer period in a carrier man without any symptom. Additional hypothesis is that since ACE2 could serve as a mediator for the endocytosis of the previously SARS coronavirus, SARS-CoV-2 may enter the cells through similar mechanism. From the protein expression atlas, high levels of ACE2 precursor were found in intestines and testis. Hence, the testis and seminal fluids could be the host cell and/or reservoir. The results could be used as a suggestive guideline for the sexual activities after the discharge or declaration of disease free.
\end{abstract}

Keywords SARS-CoV-2 $\cdot$ ACE2 $\cdot$ Semen $\cdot$ Testes $\cdot$ Seminal vesicles

\section{Perspective}

As patients with a novel coronavirus of 2019 (SARS-CoV-2) are increasing in China and other nations unexpectedly, SARS-CoV-2 has become an urgent global health concern with high number of mortality, infectivity and potential secondary/tertiary infections (Zhu et al. 2019). Currently,

Seong Soo A. An

seongaan@gachon.ac.kr

1 Department of Industrial and Environmental Engineering, Graduate School of Environment Gachon University, 1342 Sungnam-daero, Sujung-gu, Seongnam-si 461-701, Gyeonggi-do, Korea

2 Department of BionanoTechnology, Gachon Medical Research Institute, Gachon University, 1342 Sungnam-daero, Sujung-gu, Seongnam-si 461-701, Gyeonggi-do, Korea

3 Department of Internal Medicine, Yongin Severance Hospital, Yonsei University College of Medicine, 363, Dongbaekjukjeon-daero, Giheung-gu, Yongin-si 16995, Gyeonggi-do, Korea
SARS-CoV-2 could be detected in human clinical specimens of saliva, nasal fluid, blood, feces, and urine by nextgeneration sequencing, real-time RT-PCR, cell culture, and electron microscopy (Zhu et al. 2019). Importantly, recent studies indicated potential critical concerns with semen of men infected with viruses, which could be considered to be a primary route of sexually transmitted infections (Feldmann 2018) (e.g. Zika virus and Ebola virus). Hence, the semen or reproductive tracts of men should be considered for the testing of SARS-CoV-2, assessing its stabilities, half-life and infectivity.

Additional hypothesis is that since angiotensin converting enzyme 2 (ACE2) could serve as a mediator for the endocytosis of the previous coronavirus SARS crisis (Chu et al. 2005), SARS-CoV-2 may enter host cells and tissues through similar mechanism (Fig. 1). From the protein expression atlas (www.proteinatlas.org), high levels of ACE2 precursor were found in kidney, intestines and testis (Fig. 1), and not in female reproductive system, which could be the possible 


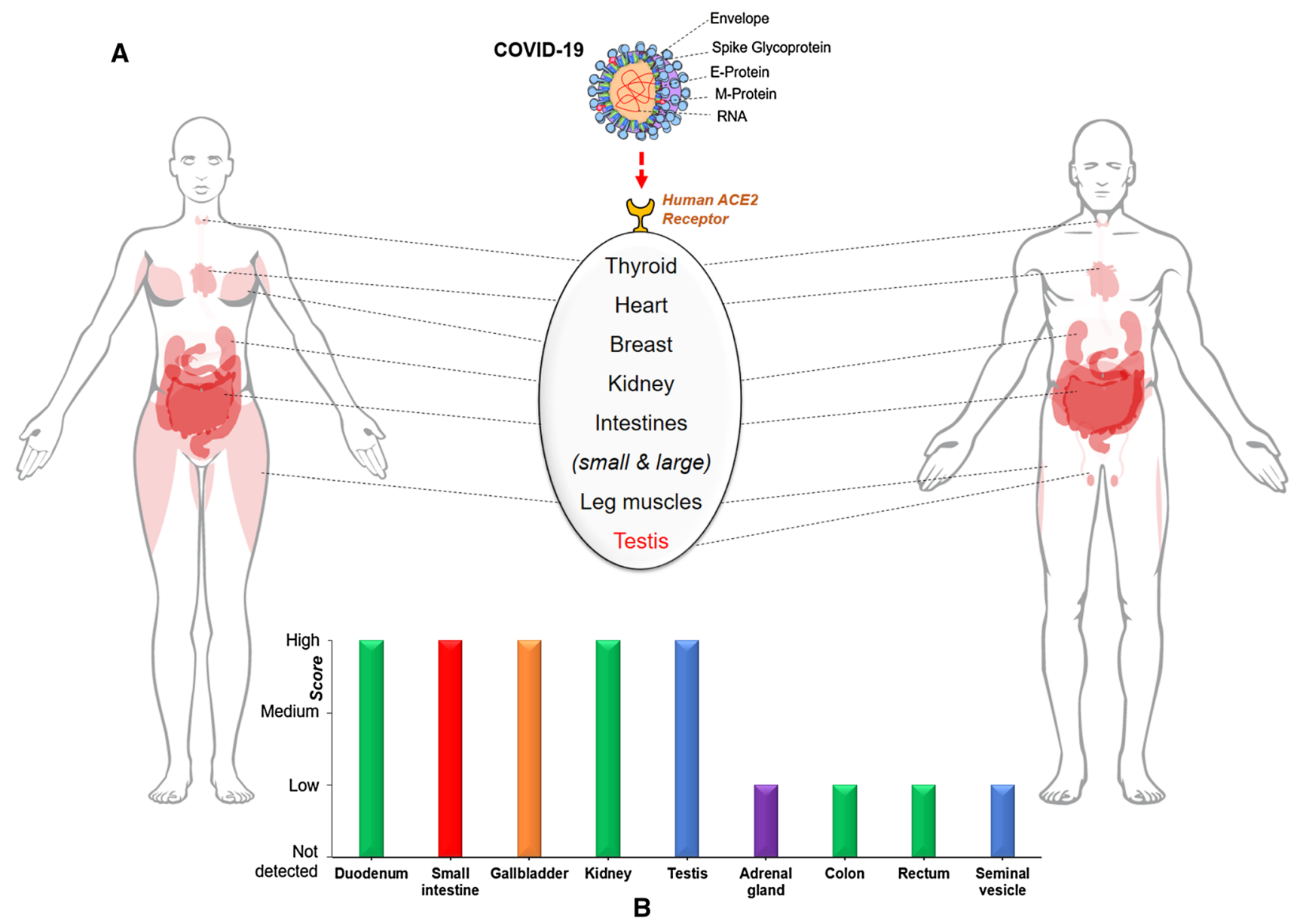

Fig. 1 a Selective ACE2 expression in thyroid, heart, breast, kidney, intestines, leg muscles and testis. b Schematic overview of the ACE2 expression is shown for each of the nine tissues. Color-coding is based on tissue groups and no protein expression data were observed in Cerebral cortex, Cerebellum, Hippocampus, Caudate, Thyroid gland, Parathyroid gland, Nasopharynx, Bronchus, Lung, Oral mucosa, Salivary gland, Esophagus, Stomach, Liver, Pancreas,

connection/explanation for the higher rate of death in men than women. (Chen et al. 2019)

ACE2 is involved in the regulation of cardiac functions and blood pressure, and its overexpression could be involved in following diseases, such as cardiac dysfunctions or diabetes. If ACE2 may participate as a receptor for SARSCoV-2, patients with hypertension, cardiac dysfunctions or diabetes could have higher risks of viral infections and faster disease progress (Chappell et al. 2014). Next, SARS$\mathrm{CoV}-2$ seemed to affect elderlies with median age of onset of 49-56 years and less prominent in children and younger populations (Rio and Malani 2019). Even though SARSCoV-2 may have similar symptoms like influenza, major difference of SARS-CoV-2 were the higher infectivity rate in elderlies with comorbidities of other diseases, while incidence in younger age groups remained lower. On the other
Urinary bladder, Epididymis, Prostate, Vagina, Ovary, Fallopian tube, Endometrium, Cervix, uterine, Placenta, Breast Heart muscle, Smooth muscle, Skeletal muscle, Soft tissue, Adipose tissue, Skin, Appendix, Spleen, Lymph node, Tonsil, Bone marrow tissues. The proteomic analysis is combined with RNA-Seq on the organ, tissue, and cellular level, and all data is freely accessible on the Human Protein Atlas web portal, www.proteinatlas.org

hand, influenza would affect both children and elderlies (Tu et al. 2020). Reduced immune system may be the highest risk factor for the onset of both SARS-CoV-2 and influenza. In elderlies and children, several innate immune functions may be reduced, resulting in higher mortality in patients with influenza (Tu et al. 2020). Higher ACE2 expressions in adults in their $40 \mathrm{~s}$ and $50 \mathrm{~s}$ may explain the higher mortality rate. And it could be possible that children are less capable for SARS-CoV-2 due to the lower expression of ACE2 (Chappell et al. 2014). Therefore, the presence or lack of ACE2 expression may be the main risk factor for viral infection or resistance.

In conclusion, the testis and seminal fluids could be the possible host and/or reservoir of SARS-CoV-2. Therefore, we would like to propose the potential testing of SARS-CoV-2 in semen for the potential early diagnostic, 
confirmatory or prognostic tests. The results could be used as a suggestive guideline for the sexual activities after the discharge or declaration of disease free.

\section{References}

Chappell MC, Marshall AC, Alzayadneh EM, Shaltout HA, Diz DI (2014) Update on the Angiotensin converting enzyme 2-Angiotensin (1-7)-MAS receptor axis: fetal programing, sex differences, and intracellular pathways. Front Endocrinol 4:201

Chen N, Zhou M, Dong X, Qu J, Gong F, Han Y, Qiu Y, Wang J, Liu Y, Wei Y, Xia J, Yu T, Zhang X, Zhang L (2020) Epidemiological and clinical characteristics of 99 cases of 2019 novel coronavirus pneumonia in Wuhan, China: a descriptive study. Lancet 395(10223):507-513

Chu KH, Tsang WK, Tang CS, Lam MF, Lai FM, To KF, Fung KS, Tang HL, Yan WW, Chan HW, Lai TS, Tong KL, Lai KN (2005)
Acute renal impairment in coronavirus-associated severe acute respiratory syndrome. Kidney Int 67(2):698-705

del Rio C, Malani PN (2020) 2019 Novel Coronavirus-important information for clinicians. JAMA 323(11):1039-1040

Feldmann H (2018) Virus in semen and the risk of sexual transmission. N Engl J Med 378(15):1440-1441

Tu H, Tu S, Gao S, Shao A, Sheng J (2020) Current epidemiological and clinical features of COVID-19; a global perspective from China. J Infect 81(1):1-9

Zhu N, Zhang D, Wang W, Li X, Yang B, Song J, Zhao X, Huang B, Shi W, Lu R, Niu P, Zhan F, Ma X, Wang D, Xu W, Wu G, Gao GF (2019) Tan W (2020) A novel coronavirus from patients with pneumonia in China. N Engl J Med 382(8):727-733

Publisher's Note Springer Nature remains neutral with regard to jurisdictional claims in published maps and institutional affiliations. 